\title{
Transfer of Gravitational Information through a Quantum Channel
}

\author{
State Key Laboratory of Magnetic Resonances and Atomic and Molecular Physics, \\ Wuhan Institute of Physics and Mathematics, \\ Chinese Academy of Sciences, Wuhan 430071, People's Republic of China
}

\begin{abstract}
Gravitational information is incorporated into an atomic state by correlation of the internal and external degrees of freedom of the atom, in the present study of the atomic interferometer. Thus it is difficult to transfer information by using a standard teleportation scheme. In this paper, we propose a novel scheme for the transfer of gravitational information through a quantum channel provided by the entangled atomic state. Significantly, the existence of a quantum channel suppresses phase noise, improving the sensitivity of the atomic interferometer. Thus our proposal provides novel readout mechanism for the interferometer with an improved signal-to-noise ratio.

Keywords: gravitational information, entanglement, interferometer

PACS: 03.67.Hk, 03.75.Dg, 04.80.-y
\end{abstract}

*Electronic address: zhangbc@wipm.ac.cn 


\section{INTRODUCTION}

One of the most important recent achievements regarding the incorporation of gravity into quantum theory, is the measurement of gravitational effects by using quantum states of microscopic particles [1 3 3 ], since gravitational forces are too weak to be readily observable on the microscopic scale. In current experiments, especially for the atomic interferometer [2, 3], the probe of gravitational effects is dependent on the change of atomic internal state, which is measured using the interference effects involving both the internal and external degrees of freedom. Moreover, due to the technological developments of laser cooling and atom trapping, more elaborate effects due to the coupling of gravity with the atomic state will be detected by the atomic interferometer in the near future [4, 5]. On the other hand, it is usually necessary to measure gravitational field in places inaccessible to people. Then, when the atomic interferometer is sent to the appointed location, we have to find a method to read the measured result. Although there might be many other methods that solve this problem, in this paper we address whether gravitational information written into the atomic state can be transferred through a quantum channel. At the same time our study also involves another problem of whether the information stored in the entangled quantum state of a single atom by correlation of its internal and external degrees of freedom (for the sake of convenience we call such a state a "specific state" in this paper) can be transferred through the quantum channel, which has not been studied up to now as far as we know.

The past few years have seen much active study of information transfer since the emergence of quantum entanglement as a channel for the transfer of information, and most of these studies focus on a method called quantum teleportation [6 8]. Thus a natural and direct thought is that whether we can use the process of quantum teleportation to implement the transfer of classical gravitational information, since this information can be stored in an atomic quantum state. Actually such a process is not feasible. The reason for this is that the atomic quantum state is formed by correlation of the internal and external degrees of freedom of a single atom that goes through the atomic interferometer. Gravitational information will be stored in the "specific state" which makes the standard teleportation process invalid since a joint measurement cannot be made in this case.

In order to realise the transfer of classical gravitational information stored in the "specific state", we propose a novel method in which two inter-entangled atoms are used, but the 
entanglement exists only between the internal atomic degrees of freedom. Thus the entangled atomic state provides a quantum channel through which gravitational information can be transferred by writing them into one part of the entangled state. However, the question of whether the existence of this quantum channel will change the sensitivity of the results for testing gravitational effects must be investigated carefully. In this paper, we put forward a practical scheme for the transfer of information, and analyze the influence of the existence of the quantum channel on the results. Our analysis reveals that the mechanism scales better with respect to phase noise than the previous one without the existence of the quantum channel. Thus our proposal provides novel readout mechanism for the interferometer, with a better signal-to-noise ratio.

The structure of this paper is as follows. We next describe how gravitational information is stored in the "specific state" in the second section. Then the third section is devoted to the investigation of the transfer of information about the classical gravitational field through quantum channel. In the fourth section, we compare the influence of noise on gravitational information for the two situations, with and without the existence of quantum channel, and find that the existence of the quantum channel improves the measurement sensitivity of the atomic interferometer. Finally, we discuss and summarise our results in the fifth section.

\section{SPECIFIC STATE}

In the above section, we have introduced the "specific state" which involves the correlation of the internal and external degrees of freedom of a single atom. In this section we will present the "specific state", without loss of generality, within the structure of the atomic interferometer proposed firstly by M. Kasevich and S. Chu [3]. Due to the absence of a complete quantum gravity theory, the interferometry process is generally described semiclassically, which means the gravitational field is considered to be classical while the motion of the matter is described according to quantum mechanics.

The interferometer considered here mainly consists of a beamsplitter-mirror-beamsplitter $\left(\frac{\pi}{2}-\pi-\frac{\pi}{2}\right)$ optical pulse sequence and is the matter-wave analog of a Mach-Zender interferometer. See Fig.1 for a description of the principle of the interferometer, which can generally be stated as such: Firstly, an atom, prepared in the ground state $|g\rangle$, interacts

with the first $\frac{\pi}{2}$ pulse, which will couple the two stable internal atomic states $|g\rangle$ and $|e\rangle$. 
Then the atomic state becomes a coherent superposition of the two states $|g\rangle$ and $|e\rangle$, with the corresponding changes to the external states, which leads to separation of the atoms on the basis of the momentum of the photon used to drive the transition. After a period of free flight of the atoms, the $\pi$ pulse forces the exchange of the internal states associated with the atomic wavepackets, and the simultaneous exchange of the external states through the photon recoil momentum. Finally, the second $\frac{\pi}{2}$ pulse induces the interference between the atomic wavepackets along different recoil paths, which leads to a change of the probability of finding the atom in the one of the two states $|g\rangle$ and $|e\rangle$, and gives the observed signal in the experiment. To sum up, we equivalently express the interference process by solving the Schrödinger equation with the following Hamiltonian [5, 9]:

$$
H=\frac{p^{2}}{2 m}+\sum_{i} E_{i}\left|A_{i}\right\rangle\left\langle A_{i}\right|-\mathbf{d} \cdot \mathbf{E}+V(x)
$$

where the first term is the kinetic energy of the atoms (which will influence the external degrees of freedom), the second term is the internal atomic structure Hamiltonian, the third term is the atom-light interaction (with $\mathbf{d}$ the electric dipole operator and $\mathbf{E}$ the electric field), and the last term describes the interaction with the gravitational field which can be expanded up to the second order as, $V(r)=V\left(r_{0}\right)-g_{a}\left(r_{a}-r_{0}\right)+\frac{1}{2} V_{a b}\left(r_{a}-r_{0}\right)\left(r_{b}-r_{0}\right)$, with the gravitational acceleration $g_{a}=-\frac{1}{m} \partial_{a} V\left(r_{0}\right)$ and the second derivative $V_{a b}=\partial_{a} \partial_{b} V\left(r_{0}\right)$ (which is related to the gravitational gradient or the Riemannian space-time curvature). Then we calculate the evolution of the state in the interaction picture, using a decomposition of the state vector of the free evolution into plane waves,

$$
|\chi\rangle=\int d \mathbf{p} \sum_{i} c_{i}(\mathbf{p}, t) e^{-i\left(E_{i}+\frac{p^{2}}{2 m}\right)\left(t-t_{0}\right)}|\mathbf{p}\rangle\left|A_{i}\right\rangle
$$

where $|\mathbf{p}\rangle$ is the eigenvector of the atom's external degrees of freedom with the momentum p. Then according to the principle of the atomic interferometer, after all interactions, we have the state

$$
|\chi\rangle=\frac{1}{2}\left[\left(1+e^{-i \Delta \phi_{t o t}}\right)|g\rangle|p\rangle+\left(1-e^{-i \Delta \phi_{t o t}}\right)|e\rangle|p+k\rangle\right]
$$

where $\Delta \phi_{\text {tot }}$ is the total phase difference [4, 5, 10 13] between two paths through the atomic interferometer, which provides the basis of an experimental observation. The state in Eq. (3) is the "specific state" that we are concerned with in this paper which can evidently be 
seen the correlation between the internal and external degrees of freedom. In addition, the state in Eq. (3) also includes information about the gravitational field around the atomic interferometer. Assuming that the duration of atom-laser interaction is short compared with the duration of the free-flight time of the atom and there is little dispersion of the atomic wavepacket in the whole interference process, the total phase difference is expressed [10, 12] as

$$
\Delta \phi_{t o t}=k g T^{2}
$$

where $k$ is the effective laser-field wavevector, $T$ is the interrogation time between two subsequent laser pulses, and $g$ is the information from the gravitational field, conditional on the experimental setup and precision. Actually, the gravitational field around the Earth is complicated and its complication has been partly observed in recent experiments. The present precision for the value of the gravitational acceleration is $\Delta g / g \simeq 3 \times 10^{-9}$, the measurement of which involved gaining information about the gravity gradient by the corresponding measurement of the phase difference [14, 15],

$$
\Delta \phi_{t o t}=\left(1+\frac{7}{12} \gamma T^{2}+\cdots\right) k g_{0} T^{2},
$$

where $\gamma \simeq 3 \times 10^{-7} \mathrm{gm}^{-1}$ is the gradient of the Earth's gravitational field. More effects due to general relativity, such as non-linear three-graviton coupling, the gravity of kinetic energy, and so on, have been analysed using the same kind of atomic interferometry and the relevant experimental setup is presently going on [5, 16]. Even information about gravitational waves from distant astrophysical events [17, 18] can also be tested using such atomic interferometry, but there is a need for higher precision and accuracy.

Finally, when the atom leaves the interferometer, the probability of finding it still in the ground state is [5, 9]

$$
P_{t}=\frac{1+\cos \left(\Delta \phi_{t o t}\right)}{2}
$$

Thus, the gravitational information is written into the atomic population, which gives the experimental signal. So the successful transfer of the classical gravitational information is equivalent to finding a similar population in a location far from the interferometer. On the other hand, it is noted that the state in Eq. (3) shows the coupling of the internal and external degrees of freedom. Thus the transfer of information is closely related to the treatment of the "specific state". 


\section{GRAVITATIONAL INFORMATION TRANSFER}

As stated in the last section, if we want to transfer classical gravitational information, the "specific state" has to be treated carefully. Due to the coupling of the internal and external degrees of freedom, in order to implement the transfer our strategy is such: At first we will prepare an entangled atomic state between only the internal degrees of freedom of two atoms, and then make one part of the entangled state go through the atomic interferometer to carry the information about the gravitational field, and make the other part maintain its initial situation. The interaction of the atom with the gravitational field leads to the coupling between its internal and external degrees of freedom, and at the same time changes the internal entanglement of the atom with the other atom; in this way gravitational information is transferred. When the part going through the interferometer finishes its interaction, we perform a velocity selection on these atoms. After all these operations, we measure the other part placed in the original location in order to to obtain the gravitational information. For clarity, see Fig.1. In what follows, we will implement this strategy.

The initial entangled atomic state prepared is

$$
|\Psi\rangle=\frac{1}{\sqrt{2}}\left(|g\rangle_{1}|e\rangle_{2}+|e\rangle_{1}|g\rangle_{2}\right)
$$

where $|g\rangle$ represents the ground state, $|e\rangle$ represents the excited state and the subscripts label the two entangled atoms. Here, we introduce a method regarding the preparation of an entangled state by the exchange of a single photon between the atoms in a high Q cavity, based on that of Ref. [19, 20]. In such a preparation the atom-cavity system undergoes oscillations, with Rabi frequency $\Omega$, between states $|g\rangle|1\rangle,|e\rangle|0\rangle$ which means that the atom in the internal state $|g\rangle$ or $|e\rangle$ coexists with one photon or zero photon in the cavity. The process can be described simply as follows: The first atom in a state $|e\rangle$ enters and couples with the cavity with a duration $\Omega t_{1}=\frac{\pi}{2}$, and then the second atom in a state $|g\rangle$ enters the cavity. Before the second atom enters, the state of the system consisting of two atoms and the cavity is

$$
\left|\Psi^{\prime}\right\rangle=\frac{1}{\sqrt{2}}\left(|g\rangle_{1}|1\rangle+|e\rangle_{1}|0\rangle\right)|g\rangle_{2}
$$

After a delay $T$, the second atom enters and couples with the cavity with a duration $\Omega t_{2}=\pi$. If the first atom has left the cavity empty, the second atom will stay in the initial state. If the first atom has emitted a photon, the second atom will absorb it and change to the state 
$|e\rangle$. As a result, the final state becomes

$$
\left|\Psi^{\prime}\right\rangle=\frac{1}{\sqrt{2}}\left(|g\rangle_{1}|e\rangle_{2}+|e\rangle_{1}|g\rangle_{2}\right)|0\rangle=|\Psi\rangle|0\rangle
$$

So the entangled atomic state appears in the presence of an empty cavity in the same form as that of Eq. (77). Notably, such an entangled atomic state has been realised experimentally with good fidelity [20, 21]. However, the entangled atomic state is fragile due to detrimental effects of the environment, so the two entangled atoms cannot be separated too far. Fortunately, a recent study regarding the dissipative preparation of entanglement in optical cavities showed that the environment can be used as a resource to help generate entanglement [22]. In the study two $\Lambda$-type three-level atoms were entangled in a detuned cavity with the interaction initiated by a off-resonance optical laser and a microwave source.

With the entangled atomic state of Eq. (7), our scheme can be implemented. Firstly we make the second atom undergo the interference process in the interferometer described in the last section, and according to the Hamiltonian in Eq. (1), the change of its internal and external atomic states is expressed as

$$
\begin{aligned}
& |g\rangle|p\rangle \rightarrow a_{1}|g\rangle|p\rangle+a_{2}|e\rangle|p+k\rangle \\
& |e\rangle|p\rangle \rightarrow b_{1}|g\rangle|p-k\rangle+b_{2}|e\rangle|p\rangle
\end{aligned}
$$

with the parameters calculated by

$$
\begin{aligned}
a_{1} & =-\frac{1}{2} e^{-i \phi_{2}+i \phi_{1}}\left(1+e^{-i \Delta \phi}\right) \\
a_{2} & =\frac{i}{2} e^{-i \phi_{2}+i \phi_{1}+i \phi_{3}}\left(1-e^{-i \Delta \phi}\right) \\
b_{1} & =\frac{i}{2} e^{i \phi_{2}-i \phi_{1}-i \phi_{3}}\left(1-e^{i \Delta \phi}\right) \\
b_{2} & =-\frac{1}{2} e^{i \phi_{2}-i \phi_{1}}\left(1+e^{i \Delta \phi}\right)
\end{aligned}
$$

where $\phi_{1}, \phi_{2}$ and $\phi_{3}$ are the initial phases of the $\frac{\pi}{2}-\pi-\frac{\pi}{2}$ laser pulse sequence, and $\Delta \phi=\left(\phi_{1}\left(t_{1}\right)-\phi_{2}\left(t_{2}\right)\right)_{\text {up-path }}-\left(\phi_{3}\left(t_{3}\right)-\phi_{2}\left(t_{2}\right)\right)_{\text {down-path }}$ where $t_{i}$ is the time of the lightatom interaction. The phase difference existing in the atomic interferometer is calculated according to the expression [4, 5, 10 12] $\Delta \phi=\Delta \phi_{\text {path }}+\Delta \phi_{\text {light }}+\Delta \phi_{\text {seperation }}$, where the phase difference $\Delta \phi_{\text {path }}$ originates from the free-flight evolution of the atom between light pulses, the phase difference $\Delta \phi_{\text {light }}$ comes from the interaction of the atom with the laser field used to manipulate the wave-function at each of the beamsplitters and mirrors in the 
interferometer, and the phase difference $\Delta \phi_{\text {seperation }}$ arises due to the final spatial separation of the interfering atomic wavepackets at the interferometer output port. Thus information about the gravitational field involved in the process is printed on the phase difference $\Delta \phi$ or the parameters $a_{1}, a_{2}, b_{1}, b_{2}$.

So if the second atom goes through the process in the atomic interferometer, after the interaction, the whole state becomes

$$
\begin{aligned}
\left|\Psi_{t}\right\rangle & =\frac{1}{\sqrt{2}}\left(|g\rangle_{1}\left(b_{1}|g\rangle_{2}|p-k\rangle+b_{2}|e\rangle_{2}|p\rangle\right)+|e\rangle_{1}\left(a_{1}|g\rangle_{2}|p\rangle+a_{2}|e\rangle_{2}|p+k\rangle\right)\right) \\
& =\frac{1}{\sqrt{2}}\left(b_{1}|g\rangle_{1}|g\rangle_{2}|p-k\rangle+\left(b_{2}|g\rangle_{1}|e\rangle_{2}+a_{1}|e\rangle_{1}|g\rangle_{2}\right)|p\rangle+a_{2}|e\rangle_{1}|e\rangle_{2}|p+k\rangle\right)
\end{aligned}
$$

When the atom leaves the interferometer, velocity selection has to be made again using the same procedure as for the atom entering into the interferometer [5, 16]. If the external state is also projected onto the eigenvector $|p\rangle$ with the same momentum $p$ as that of the entering atoms, the probability of measuring the state $|g\rangle_{1}$ is

$$
P=\frac{1+\cos (\Delta \phi)}{4}
$$

which is different from the result presented in Eq. (66). However, since the gravitational information is stored in the total phase difference $\Delta \phi$, the transfer is still complete although the observed fringe amplitude changes. Thus we realise the transportation of classical gravitational information through a quantum channel. In the following section, we will discuss the difference between the two results of Eqs. (6) and (11) and the influence of the existence of a quantum channel on the measurement sensitivity.

\section{INFLUENCE OF THE QUANTUM CHANNEL}

It is noted that gravitational information is being stored in the total phase difference $\Delta \phi$ when the information is transferred, which shows that gravitational information will not be lost in the process of transfer through the quantum channel. But the existence of the quantum channel has an influence on the measurement of gravitational information by the second atom going through the atomic interferometer, i.e. the observed fringe amplitude in

Eq. (11) becomes $\frac{1}{4}$ instead of the original amplitude $\frac{1}{2}$ in Eq. (6), given a fringe contrast of $100 \%$. So it is very interesting to see what role is played by the noise of the interferometer in the process of information transfer. Or equivalently, whether the quantum channel will 
limit the influence of noise on the measurement process, since the information about noise is also carried together with gravitational information in the transfer process. Alongside the analysis made in Ref. [13], we also carry out a simple calculation for the signal-to-noise ratio with and without the existence of the quantum channel.

Here, all we consider is the measurement of the gravitational acceleration which is expressed as

$$
\Delta \phi=\Delta \phi_{t o t}=k g T^{2}+O\left(T^{3}\right)
$$

where $O\left(T^{3}\right)$ contains the information about the higher-order correction of gravitational acceleration. Although gravitational information is stored in the total phase difference, the real measurement is of the atomic population, so we have to consider firstly the influence of change of atomic population on the results of the measurement result which is also called as shot noise. Generally, we could express the atomic population as

$$
P_{t}=\frac{1+\cos \left(\Delta \phi_{t o t}\right)}{2}=\frac{N_{t}}{N}
$$

without the existence of the quantum channel, and

$$
P=\frac{1+\cos (\Delta \phi)}{4}=\frac{N_{1}}{N}
$$

with the existence of the quantum channel, where $N_{t}$ and $N_{1}$ are the number of ground-state atoms at the end of the interrogation process for the two different situations, without and with the quantum channel, respectively. Thus assumpting a binomial distribution for the random variables, for $N$ trials and with probabilities $P_{t}$ and $P$, we estimate the shot noise contribution to each phase measurement by the formula

$$
P_{t s}=\frac{\partial \Delta \phi_{t o t}}{\partial N_{t}} \sigma_{N_{t}}=\frac{1}{\sqrt{N}}
$$

without the existence of the quantum channel, and

$$
P_{s}=\frac{\partial \Delta \phi}{\partial N_{1}} \sigma_{N_{1}}=\sqrt{\frac{2}{N}}
$$

with the existence of the quantum channel, where $\sigma_{N_{t}}$ and $\sigma_{N_{1}}$ are ordinary variances defined as $\sigma_{X}=\sqrt{\left\langle X^{2}\right\rangle-\langle X\rangle^{2}}$. Thus we find that the existence of the quantum channel will amplify the influence of shot noise on the measurement by

$$
\frac{P_{s}}{P_{t s}}=\sqrt{2} .
$$


However, in the following we find that the phase noise is suppressed by the existence of the quantum channel. Here, we analyze the phase noise using the same model in which the noise is simply regarded as proportional to the magnitude of the fluorescence signal [13, 23]. According to the model, we get

$$
\left\langle\Delta \phi_{\text {tot }}^{2}\right\rangle-\left\langle\Delta \phi_{t o t}\right\rangle^{2}=c^{2} \frac{1+\cos \left(\left\langle\Delta \phi_{t o t}\right\rangle\right)}{2}
$$

without the existence of the quantum channel, and

$$
\left\langle\Delta \phi^{2}\right\rangle-\langle\Delta \phi\rangle^{2}=c^{2} \frac{1+\cos (\langle\Delta \phi\rangle)}{4}
$$

with the existence of the quantum channel, where $c$ is a constant. Then the phase noise contribution to each phase measurement is

$$
P_{t p}=c \sin \frac{\left\langle\Delta \phi_{t o t}\right\rangle}{2}
$$

without the existence of the quantum channel, and

$$
P_{p} \simeq \frac{1}{\sqrt{2}} c \sin \frac{\langle\Delta \phi\rangle}{2}
$$

with the existence of the quantum channel, and their ratio is

$$
\frac{P_{p}}{P_{t p}} \simeq \frac{1}{\sqrt{2}} .
$$

From the comparisons of the contributions to the noise given by Eqs. (17) and (22), we find that shot noise is amplified due to the loss of atoms, but phase noise is suppressed when gravitational information is transferred through the quantum channel. It is noted that the influence on the measurement of the total phase difference of phase noise is about a hundred times larger than that of shot noise in the measurement of gravitational acceleration presented in Ref. [14], so the net result for the measurement sensitivity is that gravitational information will be affected less by noise when the quantum channel exists compared to without the existence of the quantum channel. Thus we conclude that the gravitational information transferred through the quantum channel will be purer than that measured directly, since the noise will be suppressed overall due to the existence of the quantum channel.

Then, there is still the problem of whether the quantum channel, as described by Eq. (7), is optimal for suppression of the phase noise in our scheme of information transfer. For answering this problem, we take the general entangled state 


$$
|\Phi\rangle=a|g\rangle_{1}|e\rangle_{2}+b|e\rangle_{1}|g\rangle_{2}
$$

where $|a|^{2}+|b|^{2}=1$. Then, implementing our scheme in the same way as in the above section, we obtain the final probability

$$
P_{\Phi}=\frac{1+\cos (\Delta \phi)}{2}|a|^{2} .
$$

Thus the ratio of Eq. (22) changes into

$$
\frac{P_{\Phi p}}{P_{t p}} \simeq \sqrt{|a||b|}
$$

From the perspective of suppressing phase noise, the optimal selection is $|a|=|b|=\frac{1}{\sqrt{2}}$ as found by calculating the extremum of Eq. (24) with the normalisation relation between $a$ and $b$. However, we have to investigate whether this is also optimal for the transfer of information. In order to estimate the selection for optimal transfer of information, we have to maximise the gravitational information obtained after transfer by using the entropy $S=\sum_{i} P_{i} \log P_{i}$ with the probabilities $P_{1}=\frac{1+\cos (\Delta \phi)}{2}|a|^{2}$ and $P_{2}=\frac{1-\cos (\Delta \phi)}{2}|b|^{2}$. It is not hard to find that the entropy is maximal when $|a|=|b|=\frac{1}{\sqrt{2}}$. Thus the quantum channel described by Eq. (77) is optimal not only for the suppression of the phase noise, but also for the transfer of the gravitational information.

\section{DISCUSSION AND CONCLUSION}

In this paper we propose that classical gravitational information can be transferred through a quantum channel by a different method from quantum teleportation, in which the quantum state [6] or the quantum field [8] are transferred. In our proposal, the transferred information is stored in the correlation between the internal and external degrees of freedom of a single atom. It is interesting to note that similar situations such as measurement of the gravitational constant G [24] or the general relativistic proper time [25] could be implemented with our method. Complete information transfer in our proposal is realised only by a partial measurement (sometimes this is also called a local measurement, as opposed to the non-local Bell measurement of quantum teleportation), i.e. we measure only the external state of the second atom. This partial measurement only makes the information shared between the two atoms, which is an obvious difference between our proposal and 
standard quantum teleportation. However, the information can be obtained by measuring either atom. More interesting, our present proposal also applies to the "cat state" of many atoms, i.e. the gravitational information can be read from any atom in the cat state as long as one atom of them goes through the interferometer. It is stressed that the information can only be read once even in such state of many atoms, but the potential transfer has be realised among these atoms.

Due to the existence of a quantum channel, phase noise is suppressed, and thus information transferred about the tested gravitational effects is improved in contrast to the case without the quantum channel. This is an important reason for choosing a quantum channel in our proposal, although a state without quantum correlation [26, 27], $|\phi\rangle=\frac{1}{2}\left(\left|g_{1}\right\rangle\left\langle g_{1}|\otimes| e_{2}\right\rangle\left\langle e_{2}|+| e_{1}\right\rangle\left\langle e_{1}|\otimes| g_{2}\right\rangle\left\langle g_{2}\right|\right)$, will also realise the transfer of gravitational information when the second atom goes through the interferometer. In particular, for the state $|\phi\rangle$, an interesting effect is that when the second atom finishes the interaction with the gravitational field, the new state $\left|\phi^{\prime}\right\rangle$ will include the quantum correlation between them due to the existence of cross terms such as $|g\rangle\langle e|$. So one attractive open problem is of whether gravitational interaction could generate quantum correlation for states such as $|\phi\rangle$, in contrast with the analysis in Ref. [28 -30] that suggests that gravity leads to the loss of coherence of quantum states.

In summary, firstly we presented the "specific state" in the context of the background of the atom interferometer first suggested by Kasevich and Chu, and analysed how gravitational information is stored in such a state with correlation of the internal and external degrees of freedom of the atom. Such a specific information-carrying method makes the implementation of the transfer of information by standard quantum teleportation unlikely. So in this paper we have proposed a practical and novel scheme to transfer gravitational information stored in a "specific state", and we have found that a complete transfer requires only a partial measurement. Significantly, information transferred is best with respect to phase noise for a quantum channel with maximal entanglement. Thus we have presented a novel proposal for the transfer of classical gravitational information using maximally entangled atomic pairs, which could be prepared in current experiments. In particular, due to the improved and more precise transfer of gravitational information, our proposal could also be regarded as an improved readout mechanism for an atomic interferometer. 


\section{ACKNOWLEDGEMENT}

We are grateful to the anonymous referee for their critical comments and helpful advice. This work is supported financially by National Basic Research Program of China with Grant No. 2010CB832805 and National Natural Science Foundation of China with Grant Nos. 11074283,11104324 .

[1] R. Colella, A. W. Overhauser, and S. A. Werner, Phys. Rev. Lett. 34 (1975) 1472.

[2] F. Riehle, T. Kisters, A. Witte, J. Helmcke, and C. J. Borde, Phys. Rev. Lett. 67 (1991) 177.

[3] M. A. Kasevich and S. Chu, Phys. Rev. Lett. 67 (1991) 181.

[4] S. Dimopoulos, P. W. Graham, J. M. Hogan, and M. A. Kasevich, Phys. Rev. Lett. 98 (2007) 111102.

[5] S. Dimopoulos, P. W. Graham, J. M. Hogan, and M. A. Kasevich, Phys. Rev. D 78 (2008) 042003.

[6] C. H. Bennett, G. Brassard, C. Crepeau, R. Jozsa, A. Peres, and W. K. Wootters, Phys. Rev. Lett. 70 (1993) 1895.

[7] M. A. Nielsen and I. L. Chuang, Quantum Computation and Quantum Information (Cambridge University Press, Cambridge, UK, 2000).

[8] C. Noh, A. Chia, H. Nha, M. J. Collett, and H. J. Carmichael, Phys. Rev. Lett. 102 (2009) 230501.

[9] C. Lämmerzahl, Gen. Relativ. Gravit. 28 (1996) 1043.

[10] M. A. Kasevich and S. Chu, Appl. Phys. B, 54 (1992) 321.

[11] P. Storey and C. Cohen-Tannoudji, J. Phys. France 4 (1994) 1999.

[12] A. Peters, K. Y. Chung, B. Young, J. Hensley, and S. Chu, Phil. Trans. R. Soc. Lond. A 355 (1997) 2223.

[13] U. Yurtsever, D. Strekalov, and J. P. Dowling, Eur. Phys. J. D 22, (2003) 365.

[14] A. Peters, K. Y. Chung, and S. Chu, Nature 400 (1999) 849.

[15] L. Zhou, Z. Y. Xiong, W. Yang, B. Tang, W. C. Peng, Y. B. Wang, P. Xu, J. Wang, and M. S. Zhan, Chin. Phys. Lett. 28 (2011) 013701.

[16] L. Zhou, Z. Y. Xiong, W. Yang, B. Tang, W. C. Peng, K. Hao, R. B. Li, M. Liu, J. Wang, 
and M. S. Zhan, Gen. Relativ. Gravit. 43 (2011) 1931.

[17] R. Y. Chiao and A. D. Speliotopoulos, J. Mod. Opt. 51 (2004) 861.

[18] D. F. Gao, P. Ju, B. Zhang, M. S. Zhan, Gen. Relativ. Gravit. 43 (2011) 2027.

[19] J. I. Cirac and P. Zoller, Phys. Rev. A 50 (1994) R2799.

[20] E. Hagley, X. Maitre, G. Nogues, C. Wunderlich, M. Brune, J. M. Raimond, and S. Haroche, Phys. Rev. Lett. 79 (1997) 1.

[21] A. Rauschenbeutel, G. Nogues, S. Osnaghi, P. Bertet, M. Brune, J. -M. Raimond, and S. Haroche, Science 288 (2000) 2024.

[22] M. J. Kastoryano, F. Reiter, and A. S. Sorensen, Phys. Rev. Lett. 106 (2011) 090502.

[23] M. J. Snadden, J. M. McGuirk, P. Bouyer, K. G. Haritos, and M. A. Kasevich, Phys. Rev. Lett. 81 (1998) 971.

[24] J. B. Fixler, G. T. Foster, J. M. McGuirk, and M. A. Kasevich, Science 315, (2007) 74.

[25] M. Zych, F. Costa, L. Pikovski, C. Brukner, Nature Communication 2 (2011) 505.

[26] M. Horodecki, P. Horodecki, R. Horodecki, J. Oppenheim, A. Sen, U. Sen, and B. SynakRadtke, Phys. Rev. A 71 (2005) 062307.

[27] A. Streltsov, H. Kampermann, and D. Bruß, Phys. Rev. Lett. 107 (2011) 170502.

[28] L. Diósi, Phys. Lett. A 105A (1984) 199.

[29] R. Penrose, Gen. Relativ. Gravit. 28 (1996) 581.

[30] S. L. Adler, J. Phys. A: Math. Theor. 40 (2007) 755. 


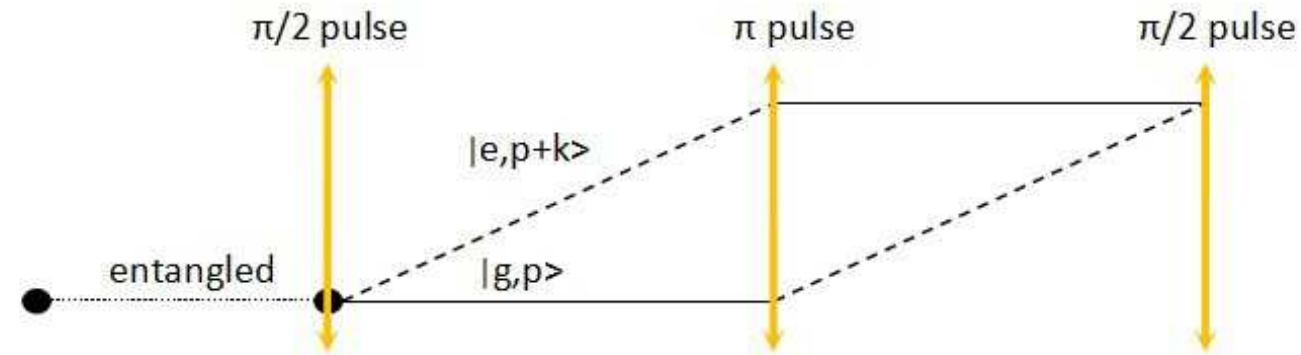

FIG. 1: Sketch of our scheme. The left sector is the quantum channel provided by the most entangled atomic pairs. The right sector is the schematic of the atomic interferometer. 